\title{
Simplified Method of Conjugation of Silver Nanoparticles to Type I Collagen: Morphological Evidence for a Stable Microparticle
}

\author{
W. J. L'Amoreaux ${ }^{*}$, K. Campbell ${ }^{* *}$, R. Sardar ${ }^{* * *}$, and B. P. S. Chauhan ${ }^{* * *}$ \\ *Department of Biology, The City University of New York, College of Staten Island, \\ 2800 Victory Blvd., Staten Island, NY 10314 \\ ** Department of Biomedical Engineering, University of Wisconsin at Madison, Room \\ 2681410 Engineering Drive Madison, WI 53706-1608 \\ *** Department of Chemistry, The City University of New York, College of Staten \\ Island, 2800 Victory Blvd., Staten Island, NY 10314
}

Drug delivery and targeting on the molecular level comprises a fast growing branch of the biomaterials and bioengineering fields. A requirement for success is the proper choice of biomaterial used. Currently, there are several polymers used as biomaterials, many of which can cause long-term side effects and complications ${ }^{1}$. Collagen is a natural, inert biomaterial found suitable for drug delivery. It is valued for its superior biocompatibility, biodegradability, and weak antigenicity relative to other polymers used as biomaterials ${ }^{2}$. It has been used in resorbable surgical sutures, hemostatic agents, and wound dressings for many years and shows promise for future development in the drug delivery field ${ }^{3}$. Collagen has been rarely implicated in adverse reactions and can be molded into a variety of drug delivery systems ${ }^{2}$.

The silver nanoparticles generated in this study were synthesized according to a technique previously described ${ }^{5}$. The suspension of 1 eq. of silver acetate (AgOAc) was prepared in distilled benzene. After 5 min. of constant stirring, 2 eq. PMHS (poly-methyl-hydrosiloxane) were added to the AgOAc solution. Following AgOAc preparation, 6 eq of capping agent (APTS) were dissolved in benzene. The final product contained a silver concentration of $320 \mu \mathrm{g} / \mathrm{ml}$. The stock collagen used was type I rat tail collagen (CI) (Becton-Dickenson) at a concentration of $4.31 \mathrm{mg} / \mathrm{ml}$ in acetic acid. The collagen was then diluted with acetic acid to a concentration of 100 $\mu \mathrm{g} / \mathrm{ml}$. The collagen samples were combined with the nanoparticles by adding the silver acetate solution drop wise to the $\mathrm{CI}$. These $\mathrm{Ag} / \mathrm{CI}$ solutions were then allowed to incubate for 18 hours at $4^{\circ} \mathrm{C}$. The $\mathrm{Ag} / \mathrm{CI}$ copolymers were imaged by transmission and scanning electron microscopy to determine the extent of silver incorporation and to determine if morphological changes in the collagen biopolymers occurred.

As was expected, type I collagen in its native state produced long fibrils measuring $\sim 20 \mathrm{~nm}$ in diameter (Figures 1,2). For collagen incubated in the presence of silver acetate, silver nanoparticles were observed in tightly packed copolymers (Figures 3 6). Our morphological characterization demonstrates that the addition of silver caused no deleterious effects on the collagen fibers. In the collagen samples without silver, there more amorphous, web-like arrangements of collagen, while in those samples with silver display a more ordered array of fibers. In conclusion, this study showed that it is possible to create collagen - silver microparticles from soluble 
collagen. The data suggest that the silver nanoparticles were nucleated by the collagen fibers, which caused a morphology change in the fibers.
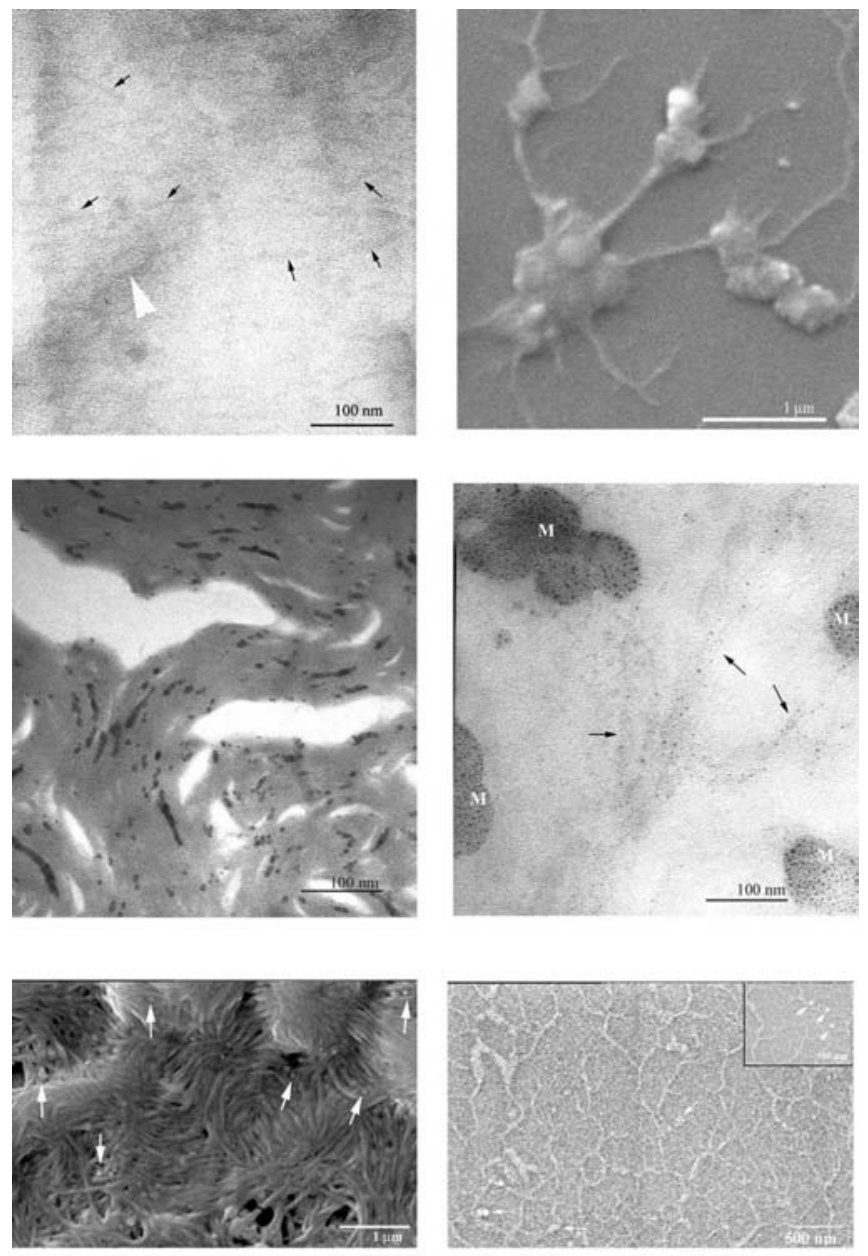

Figure 1. TEM of type I collagen. Individual fibrils (arrows) and an aggregate of fibers (arrowhead) were observed.

Figure 2. SEM of type I collagen. Individual fibrils (arrows) as well as aggregates of collagen were observed.

Figure 3. TEM of type I collagen plus silver acetate. Individual fibrils were observed and silver incorporation was abundant. The fibers were more tightly packed.

Figure 4. TEM of type I collagen plus silver acetate. Individual fibrils were observed and silver incorporation was abundant. The fibers were not as nearly packed as in Figure 3. Silver acetate micelles can also be observed (M).

Figure 5. SEM of type I collagen plus silver acetate. Individual fibrils were observed and silver incorporation was abundant. The fibers were tightly packed, confirming our observation using TEM.

Figure 6. SEM of type I collagen plus silver acetate. Individual fibrils were observed but silver incorporation was less abundant than in Figure 5. Inset: higher resolution of silver-collagen copolymer. 